\title{
A Set of Mathematical Constants Arising Naturally in the Theory of the Multiple Gamma Functions
}

\author{
Junesang Choi \\ Department of Mathematics, Dongguk University, Gyeongju 780-714, Republic of Korea \\ Correspondence should be addressed to Junesang Choi, junesang@mail.dongguk.ac.kr
}

Received 7 August 2012; Accepted 10 September 2012

Academic Editor: Sung G. Kim

Copyright (C) 2012 Junesang Choi. This is an open access article distributed under the Creative Commons Attribution License, which permits unrestricted use, distribution, and reproduction in any medium, provided the original work is properly cited.

We introduce a set of mathematical constants which is involved naturally in the theory of multiple Gamma functions. Then we present general asymptotic inequalities for these constants whose special cases are seen to contain all results very recently given in Chen 2011.

\section{Introduction and Preliminaries}

The double Gamma function $\Gamma_{2}=1 / G$ and the multiple Gamma functions $\Gamma_{n}$ were defined and studied systematically by Barnes [1-4] in about 1900. Before their investigation by Barnes, these functions had been introduced in a different form by, for example, Hölder [5], Alexeiewsky [6] and Kinkelin [7]. In about the middle of the 1980s, these functions were revived in the study of the determinants of the Laplacians on the $n$-dimensional unit sphere $\mathbf{S}^{n}$ (see [8-13]). Since then the multiple Gamma functions have attracted many authors' concern and have been used in various ways. It is seen that a set of constants $\left\{A_{q} \mid q \in \mathbb{N}:=\right.$ $\{1,2,3, \ldots\}\}$ given in (1.11) involves naturally in the theory of the multiple Gamma functions $\Gamma_{n}$ (see [14-20] and references therein). For example, for sufficiently large real $x$ and $a \in \mathbb{C}$, we have the Stirling formula for the G-function (see [1]; see also [21, page 26, equation (7)]):

$$
\begin{aligned}
\log G(x+a+1)= & \frac{x+a}{2} \log (2 \pi)-\log A+\frac{1}{12}-\frac{3 x^{2}}{4}-a x \\
& +\left(\frac{x^{2}}{2}-\frac{1}{12}+\frac{a^{2}}{2}+a x\right) \log x+O\left(x^{-1}\right) \quad(x \longrightarrow \infty),
\end{aligned}
$$


where $A$ is the Glaisher-Kinkelin constant (see [7, 22-24]) given in (1.16) below. The GlaisherKinkelin constant $A$, the constants $B$ and $C$ below introduced by Choi and Srivastava have been used, among other things, in the closed-form evaluation of certain series involving zeta functions and in calculation of some integrals of multiple Gamma functions. So trying to give asymptotic formulas for these constants $A, B$, and $C$ are significant. Very recently Chen [25] presented nice asymptotic inequalities for these constants $A, B$, and $C$ by mainly using the Euler-Maclaurin summation formulas. Here, we aim at presenting asymptotic inequalities for a set of the mathematical constants $A_{q}(q \in \mathbb{N})$ given in (1.11) some of whose special cases are seen to yield all results in [25].

For this purpose, we begin by summarizing some differential and integral formulas of the function $f(x)$ in (1.2).

Lemma 1.1. Differentiating the function

$$
f(x):=x^{q} \log x \quad(q \in \mathbb{N} ; x>0)
$$

$\ell$ times, we obtain

$$
f^{(\ell)}(x)=x^{q-\ell}\left\{\prod_{j=1}^{\ell}(q-j+1) \log x+P_{\ell}(q)\right\} \quad(\ell \in \mathbb{N} ; 1 \leqq \ell \leqq q),
$$

where $P_{\ell}(q)$ is a polynomial of degree $\ell-1$ in $q$ satisfying the following recurrence relation:

$$
P_{\ell}(q)= \begin{cases}(q-\ell+1) P_{\ell-1}(q)+\prod_{j=1}^{\ell-1}(q-j+1) & (\ell \in \mathbb{N} \backslash\{1\} ; 2 \leqq \ell \leqq q) \\ 1 & (\ell=1)\end{cases}
$$

In fact, by mathematical induction on $\ell \in \mathbb{N}$, we can give an explicit expression for $P_{\ell}(q)$ as follows:

$$
P_{\ell}(q)=\prod_{j=1}^{\ell}(q-j+1) \cdot \sum_{j=1}^{\ell} \frac{1}{q-j+1} \quad(\ell \in \mathbb{N} ; 1 \leqq \ell \leqq q) .
$$

Setting $\ell=q$ in (1.3) and (1.5), respectively, we get

$$
f^{(q)}(x)=q !\left(\log x+H_{q}\right) \quad(q \in \mathbb{N})
$$

where $H_{n}$ are the harmonic numbers defined by

$$
H_{n}:=\sum_{j=1}^{n} \frac{1}{j} \quad(n \in \mathbb{N})
$$


Differentiating $f^{(q)}(x)$ in (1.6) $r$ times, we obtain

$$
f^{(q+r)}(x)=(-1)^{r+1} q !(r-1) ! \frac{1}{x^{r}} \quad(r \in \mathbb{N}) .
$$

Integrating the function $f(x)$ in (1.2) from 1 to $n$, we get

$$
\int_{1}^{n} f(x) d x=\frac{n^{q+1}}{q+1} \log n+\frac{1-n^{q+1}}{(q+1)^{2}} \quad(q, n \in \mathbb{N}) .
$$

For each $q \in \mathbb{N}$, define a sequence $\left\{A_{q}(n)\right\}_{n=1}^{\infty}$ by

$$
\begin{aligned}
\log A_{q}(n):= & \sum_{k=1}^{n} k^{q} \log k \\
& -\left(\frac{n^{q+1}}{q+1}+\frac{n^{q}}{2}+\sum_{r=1}^{[(q+1) / 2]} \frac{B_{2 r}}{(2 r) !} \cdot \prod_{j=1}^{2 r-1}(q-j+1) \cdot n^{q+1-2 r}\right) \log n \\
& +\frac{n^{q+1}}{(q+1)^{2}}-\sum_{r=1}^{[(q+1) / 2]+\left((-1)^{q}-1\right) / 2} \frac{B_{2 r}}{(2 r) !} P_{2 r-1}(q) n^{q+1-2 r} \quad(n, q \in \mathbb{N}),
\end{aligned}
$$

where $B_{r}$ are Bernoulli numbers given in (1.12), $P_{r}(q)$ are given in (1.5), and $[x]$ denotes (as usual) the greatest integer $\leqq x$. Define a set of mathematical constants $A_{q}(q \in \mathbb{N})$ by

$$
\log A_{q}:=\lim _{n \rightarrow \infty} \log A_{q}(n) \quad(q \in \mathbb{N}) .
$$

The Bernoulli numbers $B_{r}$ are defined by the generating function (see [21, Section 1.6]; see also, [26, Section 1.7]):

$$
\frac{z}{e^{z}-1}=\sum_{r=0}^{\infty} B_{r} \frac{z^{r}}{r !} \quad(|z|<2 \pi)
$$

We introduce a well-known formula (see [21, Section 2.3]):

$$
B_{2 p}=(-1)^{p+1} \frac{2(2 p) !}{(2 \pi)^{2 p}} \zeta(2 p) \quad\left(p \in \mathbb{N}_{0}:=\mathbb{N} \cup\{0\}\right),
$$

where $\zeta(s)$ is the Riemann Zeta function defined by

$$
\zeta(s):= \begin{cases}\sum_{n=1}^{\infty} \frac{1}{n^{s}}=\frac{1}{1-2^{-s}} \sum_{n=1}^{\infty} \frac{1}{(2 n-1)^{s}} & (\mathfrak{R}(s)>1) \\ \frac{1}{1-2^{1-s}} \sum_{n=1}^{\infty} \frac{(-1)^{n-1}}{n^{s}} & (\mathfrak{R}(s)>0 ; s \neq 1) .\end{cases}
$$


It is easy to observe from (1.13) that

$$
B_{4 p}<0, \quad B_{4 p+2}>0 \quad\left(p \in \mathbb{N}_{0}\right)
$$

Remark 1.2. We find that the constants $A_{1}, A_{2}$ and $A_{3}$ correspond with the Glaisher-Kinkelin constant $A$, the constants $B$ and $C$ introduced by Choi and Srivastava, respectively:

$$
\log A_{1}=\lim _{n \rightarrow \infty}\left\{\sum_{k=1}^{n} k \log k-\left(\frac{n^{2}}{2}+\frac{n}{2}+\frac{1}{12}\right) \log n+\frac{n^{2}}{4}\right\}=\log A
$$

where $A$ denotes the Glaisher-Kinkelin constant whose numerical value is

$$
\begin{gathered}
A \cong 1.282427130 \cdots, \\
\log A_{2}=\lim _{n \rightarrow \infty}\left\{\sum_{k=1}^{n} k^{2} \log k-\left(\frac{n^{3}}{3}+\frac{n^{2}}{2}+\frac{n}{6}\right) \log n+\frac{n^{3}}{9}-\frac{n}{12}\right\}=\log B \\
\log A_{3}=\lim _{n \rightarrow \infty}\left\{\sum_{k=1}^{n} k^{3} \log k-\left(\frac{n^{4}}{4}+\frac{n^{3}}{2}+\frac{n^{2}}{4}-\frac{1}{120}\right) \log n+\frac{n^{4}}{16}-\frac{n^{2}}{12}\right\}=\log C
\end{gathered}
$$

Here $B$ and $C$ are constants whose approximate numerical values are given by

$$
B \cong 1.03091675 \cdots, \quad C \cong 0.97955746 \cdots .
$$

The constants $B$ and $C$ were considered recently by Choi and Srivastava $[16,18]$. See also Adamchik [27, page 199]. Bendersky [28] presented a set of constants including $B$ and $C$.

\section{Euler-Maclaurin Summation Formula}

We begin by recalling the Euler-Maclaurin summation formula ( $f f$. Hardy ([29, 30], page 318)):

$$
\sum_{k=1}^{n} f(k) \sim C_{0}+\int_{a}^{n} f(x) d x+\frac{1}{2} f(n)+\sum_{r=1}^{\infty} \frac{B_{2 r}}{(2 r) !} f^{(2 r-1)}(n)
$$

where $C_{0}$ is an arbitrary constant to be determined in each special case and $B_{r}$ are the Bernoulli numbers given in (1.12). For another useful summation formula, see Edwards [31, page 117]. 
Let $f$ be a function of class $C^{2 p+2}([a, b])$, and let the interval $[a, b]$ be partitioned into $m$ subintervals of the same length $h=(b-a) / m$. Then we have another useful form of the Euler-Maclaurin summation formula (see, e.g., [32]): There exists $0<\theta<1$ such that

$$
\begin{aligned}
\sum_{k=0}^{m} f(a+k h)= & \frac{1}{h} \int_{a}^{b} f(x) d x+\frac{f(a)+f(b)}{2} \\
& +\sum_{k=1}^{p} \frac{h^{2 k-1}}{(2 k) !} B_{2 k}\left(f^{(2 k-1)}(b)-f^{(2 k-1)}(a)\right) \\
& +\frac{h^{2 p+2}}{(2 p+2) !} B_{2 p+2} \sum_{k=0}^{m-1} f^{(2 p+2)}(a+k h+\theta h),
\end{aligned}
$$

where $m, p \in \mathbb{N}$. Under the same conditions in (2.2), setting $m=n-1, a=1, b=n$, and $h=1$ in (2.2), we obtain a simple summation formula (see [25]):

$$
\sum_{k=1}^{m} f(k)=\int_{1}^{n} f(x) d x+\frac{f(1)+f(n)}{2}+\sum_{k=1}^{p} \frac{B_{2 k}}{(2 k) !}\left(f^{(2 k-1)}(n)-f^{(2 k-1)}(1)\right)+R_{n}(f, p),
$$

where, for convenience, the remainder term $R_{n}(f, p)$ is given by

$$
R_{n}(f, p):=\frac{B_{2 p+2}}{(2 p+2) !} \sum_{k=1}^{n-1} f^{(2 p+2)}(k+\theta)
$$

which is seen to be bounded by

$$
\left|R_{n}(f, p)\right| \leqq \frac{2}{(2 \pi)^{2 p}} \int_{1}^{n}\left|f^{(2 p+1)}(x)\right| d x
$$

Zhu and Yang [33] established some useful formulas originated from the EulerMaclaurin summation formula (2.1) (see also [25]) asserted by the following lemma.

Lemma 2.1. Let $\ell \in \mathbb{N}$ and let $f$ have its first $2 p+2$ derivatives on an interval $[\ell, \infty)$ such that $f^{(2 p)}(x)>0$ and $f^{(2 p+2)}(x)>0\left(\right.$ or $f^{(2 p)}(x)<0$ and $\left.f^{(2 p+2)}(x)<0\right)$ and $f^{(2 p-1)}(\infty)=0$. Then the following results hold true:

(i) The sequence

$$
\mathbf{a}_{\mathbf{n}}:=\sum_{k=\ell}^{n} f(k)-\int_{\ell}^{n} f(x) d x-\frac{1}{2} f(n)-\sum_{k=1}^{p-1} \frac{B_{2 k}}{(2 k) !} f^{(2 k-1)}(n) \quad(n \geqq \ell)
$$

converges. Let $\mathbf{a}:=\lim _{n \rightarrow \infty} \mathbf{a}_{\mathbf{n}}$. 
(ii) For $f^{(2 p)}(x)>0$ and $f^{(2 p+2)}(x)>0$, we have

$$
0<(-1)^{p-1}\left(\mathbf{a}-\mathbf{a}_{\mathbf{n}}\right)<(-1)^{p} \frac{B_{2 p}}{(2 p) !} f^{(2 p-1)}(n) \quad(n \geqq \ell) .
$$

For $f^{(2 p)}(x)<0$ and $f^{(2 p+2)}(x)<0$, we have

$$
0>(-1)^{p-1}\left(\mathbf{a}-\mathbf{a}_{\mathbf{n}}\right)>(-1)^{p} \frac{B_{2 p}}{(2 p) !} f^{(2 p-1)}(n) \quad(n \geqq \ell) .
$$

(iii) There exists $\theta \in(0,1)$ such that

$$
\sum_{k=\ell}^{n} f(k)=\mathbf{a}+\int_{\ell}^{n} f(x) d x+\frac{1}{2} f(n)+\sum_{k=1}^{p-1} \frac{B_{2 k}}{(2 k) !} f^{(2 k-1)}(n)+\theta \cdot \frac{B_{2 p}}{(2 p) !} f^{(2 p-1)}(n)
$$

\section{Asymptotic Formulas and Inequalities for $A_{q}$}

Applying the function $f(x)$ in (1.2) to the Euler-Maclaurin summation formula (2.1) with $a=1$ and using the results presented in Lemma 1.1, we obtain an asymptotic formula for the sequence $A_{q}(n)$ as in the following theorem.

Theorem 3.1. The following asymptotic formulas for the constants $A_{q}(n)$ and $A_{q}$ hold true:

$$
\begin{aligned}
\log A_{q}(n) \sim C_{q} & +\frac{1}{(q+1)^{2}}+\frac{1-(-1)^{q}}{2} \frac{B_{q+1} H_{q}}{q+1} \\
& +(-1)^{q} q ! \sum_{r=[(q+1) / 2]+1}^{\infty} \frac{B_{2 r}}{(2 r) !} \frac{(2 r-q-2) !}{n^{2 r-q-1}}
\end{aligned}
$$

where $C_{q}$ 's are constants dependent on each $q$ and an empty sum is understood (as usual) to be nil. And

$$
\log A_{q}=\lim _{n \rightarrow \infty} \log A_{q}(n)=C_{q}+\frac{1}{(q+1)^{2}}+\frac{1-(-1)^{q}}{2} \frac{B_{q+1} H_{q}}{q+1} .
$$

Proof. We only note that

(i) $1 \leqq r \leqq[(q+1) / 2]$

$$
f^{(2 r-1)}(n)=n^{q+1-2 r} \cdot \prod_{j=1}^{2 r-1}(q-j+1) \cdot \log n+n^{q+1-2 r} P_{2 r-1}(q) .
$$


Abstract and Applied Analysis

(ii) $r \geqq[(q+1) / 2]+1$

$$
f^{(2 r-1)}(n)=(-1)^{q} q ! \frac{(2 r-q-2) !}{n^{2 r-q-1}} .
$$

Applying the function $f(x)$ in (1.2) to the formula (2.9) with $\ell=1$, and using the results presented in Lemma 1.1, we get two sided inequalities for the difference of $\log A_{q}(n)$ and $\log A_{q}$ asserted by Theorem 3.2.

Theorem 3.2. The following inequalities hold true:

$$
\begin{aligned}
q ! & \sum_{r=[(q+1) / 2]+1}^{2 p} \frac{(2 r-q-2) !}{(2 r) !} \frac{B_{2 r}}{n^{2 r-q-1}} \\
& <(-1)^{q}\left(\log A_{q}(n)-\log A_{q}\right) \\
& <q ! \sum_{r=[(q+1) / 2]+1}^{2 p+1} \frac{(2 r-q-2) !}{(2 r) !} \frac{B_{2 r}}{n^{2 r-q-1}} \quad(p, q, n \in \mathbb{N}) .
\end{aligned}
$$

Proof. Setting the function $f(x)$ in (1.2) in the formula (2.9) with $\ell=1$, and using the results presented in Lemma 1.1, we get

$$
\begin{aligned}
\log A_{q}(n)= & \log A_{q}+(-1)^{q} q ! \sum_{r=[(q+1) / 2]+1}^{p-1} \frac{(2 r-q-2) !}{(2 r) !} \frac{B_{2 r}}{n^{2 r-q-1}} \\
& +(-1)^{q} q ! \frac{(2 p-q-2) !}{(2 p) !} \frac{B_{2 p}}{n^{2 p-q-1}} \theta
\end{aligned}
$$

for some $\theta \in(0,1)$.

Replacing $p$ by $2 p+1$ and $2 p+2$ in (3.6), respectively, we obtain

$$
\begin{aligned}
\log A_{q}(n)-\log A_{q}= & (-1)^{q} q ! \sum_{r=[(q+1) / 2]+1}^{2 p} \frac{(2 r-q-2) !}{(2 r) !} \frac{B_{2 r}}{n^{2 r-q-1}} \\
& +(-1)^{q} q ! \frac{(4 p-q) !}{(4 p+2) !} \frac{B_{4 p+2}}{n^{4 p+1-q}} \theta . \\
\log A_{q}(n)-\log A_{q}= & (-1)^{q} q ! \sum_{r=[(q+1) / 2]+1}^{2 p+1} \frac{(2 r-q-2) !}{(2 r) !} \frac{B_{2 r}}{n^{2 r-q-1}} \\
& +(-1)^{q} q ! \frac{(4 p+2-q) !}{(4 p+4) !} \frac{B_{4 p+4}}{n^{4 p+3-q}} \theta .
\end{aligned}
$$


In view of (1.15), we find the following inequalities:

$$
\begin{aligned}
q ! & \sum_{r=[(q+1) / 2]+1}^{2 p} \frac{(2 r-q-2) !}{(2 r) !} \frac{B_{2 r}}{n^{2 r-q-1}} \\
& <\log A_{q}(n)-\log A_{q} \\
& <q ! \sum_{r=[(q+1) / 2]+1}^{2 p+1} \frac{(2 r-q-2) !}{(2 r) !} \frac{B_{2 r}}{n^{2 r-q-1}} \quad(q \text { is even }), \\
q ! \sum_{r=[(q+1) / 2]+1}^{2 p} \frac{(2 r-q-2) !}{(2 r) !} \frac{B_{2 r}}{n^{2 r-q-1}} & \\
& <\log A_{q}-\log A_{q}(n) \\
& <q ! \sum_{r=[(q+1) / 2]+1}^{2 p+1} \frac{(2 r-q-2) !}{(2 r) !} \frac{B_{2 r}}{n^{2 r-q-1}} \quad(q \text { is odd }) .
\end{aligned}
$$

Finally it is easily seen that the two-sided inequalities (3.8) can be expressed in a single form (3.5).

Remark 3.3. The special cases of (3.5) when $q=1, q=2$, and $q=3$ are easily seen to correspond with Equations (8), (31), and (32) in Chen's work [25], respectively.

Applying the function $f(x)$ in (1.2) to the formula (2.3) and using the results presented in Lemma 1.1, we get two-sided inequalities for the $\log A_{q}$ asserted by Theorem 3.4.

Theorem 3.4. The following inequalities hold true:

$$
\begin{aligned}
\alpha_{q}+q ! & \sum_{k=[(q+1) / 2]+1}^{2 p} \frac{(2 k-q-2) !}{(2 k) !} B_{2 k} \\
& <\log A_{q} \\
& <\alpha_{q}+q ! \sum_{k=[(q+1) / 2]+1}^{2 p+1} \frac{(2 k-q-2) !}{(2 k) !} B_{2 k} \quad(q \text { is odd }), \\
\alpha_{q}-q ! & \sum_{k=[(q+1) / 2]+1}^{2 p+1} \frac{(2 k-q-2) !}{(2 k) !} B_{2 k} \\
& <\log A_{q} \sum_{k=[(q+1) / 2]+1} \frac{(2 k-q-2) !}{(2 k) !} B_{2 k} \quad(q \text { is even }),
\end{aligned}
$$


where, for convenience,

$$
\alpha_{q}:=\frac{1}{(q+1)^{2}}+\frac{1-(-1)^{q}}{2} \frac{B_{q+1} H_{q}}{q+1}-\sum_{k=1}^{[(q+1) / 2]} \frac{B_{2 k}}{(2 k) !} P_{2 k-1}(q) \quad(q \in \mathbb{N})
$$

Proof. Setting the function $f(x)$ in (1.2) in the formula (2.3), and using the results presented in Lemma 1.1, we have, for some $\theta \in(0,1)$,

$$
\begin{aligned}
\log A_{q}(n)= & \alpha_{q}+(-1)^{q} q ! \sum_{k=[(q+1) / 2]+1}^{p} \frac{(2 k-2-q) !}{(2 k) !} B_{2 k}\left(\frac{1}{n^{2 k-1-q}}-1\right) \\
& +(-1)^{q+1} q ! \frac{(2 p+1-q) !}{(2 p+2) !} B_{2 p+2} \sum_{k=1}^{n-1} \frac{1}{(k+\theta)^{2 p+2-q}} .
\end{aligned}
$$

Replacing $p$ by $2 p$ and $2 p+1$ in (3.12), respectively, we obtain

$$
\begin{aligned}
\log A_{q}(n)= & \alpha_{q}+(-1)^{q} q ! \sum_{k=[(q+1) / 2]+1}^{2 p} \frac{(2 k-2-q) !}{(2 k) !} B_{2 k}\left(\frac{1}{n^{2 k-1-q}}-1\right) \\
& +(-1)^{q+1} q ! \frac{(4 p+1-q) !}{(4 p+2) !} B_{4 p+2} \sum_{k=1}^{n-1} \frac{1}{(k+\theta)^{4 p+2-q}}, \\
\log A_{q}(n)= & \alpha_{q}+(-1)^{q} q ! \sum_{k=[(q+1) / 2]+1}^{2 p+1} \frac{(2 k-2-q) !}{(2 k) !} B_{2 k}\left(\frac{1}{n^{2 k-1-q}}-1\right) \\
& +(-1)^{q+1} q ! \frac{(4 p+3-q) !}{(4 p+4) !} B_{4 p+4} \sum_{k=1}^{n-1} \frac{1}{(k+\theta)^{4 p+4-q}} .
\end{aligned}
$$

In view of (1.15), we find from (3.13) that

$$
\begin{aligned}
\alpha_{q}-q ! & \sum_{k=[(q+1) / 2]+1}^{2 p} \frac{(2 k-2-q) !}{(2 k) !} B_{2 k}\left(\frac{1}{n^{2 k-1-q}}-1\right) \\
& <\log A_{q}(n) \\
& <\alpha_{q}-q ! \sum_{k=[(q+1) / 2]+1}^{2 p+1} \frac{(2 k-2-q) !}{(2 k) !} B_{2 k}\left(\frac{1}{n^{2 k-1-q}}-1\right) \quad(q \text { is odd }),
\end{aligned}
$$




$$
\begin{aligned}
\alpha_{q}+q ! & \sum_{k=[(q+1) / 2]+1}^{2 p+1} \frac{(2 k-2-q) !}{(2 k) !} B_{2 k}\left(\frac{1}{n^{2 k-1-q}}-1\right) \\
& <\log A_{q}(n) \\
& <\alpha_{q}+q ! \sum_{k=[(q+1) / 2]+1}^{2 p} \frac{(2 k-2-q) !}{(2 k) !} B_{2 k}\left(\frac{1}{n^{2 k-1-q}}-1\right) \quad(q \text { is even }) .
\end{aligned}
$$

Now, taking the limit on each side of the inequalities in (3.14) as $n \rightarrow \infty$, we obtain the results in Theorem 3.4.

Remark 3.5. It is easily seen that the specialized inequalities of (3.9) when $q=1$ and $q=3$ and (3.10) when $q=2$ correspond with those inequalities of Equations (9), (34), and (33) in Chen's work [25], respectively.

\section{Acknowledgments}

This research was supported by Basic Science Research Program through the National Research Foundation of Korea funded by the Ministry of Education, Science and Technology (2012-0002957).

\section{References}

[1] E. W. Barnes, "The theory of the G-function," Quarterly Journal of Mathematics, vol. 31, pp. 264-314, 1899.

[2] E. W. Barnes, "The genesis of the double gamma functions," Proceedings of the London Mathematical Society, vol. 31, no. 1, pp. 358-381, 1899.

[3] E. W. Barnes, "The theory of the double Gamma function," Philosophical Transactions of the Royal Society A, vol. 196, pp. 265-388, 1901.

[4] E. W. Barnes, "On the theory of the multiple Gamma functions," Transactions of the Cambridge Philosophical Society, vol. 19, pp. 374-439, 1904.

[5] O. Hölder, Uber Eine Transcendente Funktion, vol. 1886, Dieterichsche, Göttingen, Germany, 1886.

[6] W. P. Alexeiewsky, Uber Eine Classe von Funktionen, die der Gammafunktion Analog Sind, vol. 46, Leipzig Weidmannsche Buchhandlung, 1894.

[7] V. H. Kinkelin, "Uber eine mit der Gamma Funktion verwandte transcendente und deren Anwendung auf die integralrechnung," Journal für Die Reine und Angewandte Mathematik, vol. 57, pp. $122-158,1860$.

[8] J. Choi, “Determinant of Laplacian on $S^{3}$," Mathematica Japonica, vol. 40, no. 1, pp. 155-166, 1994.

[9] H. Kumagai, "The determinant of the Laplacian on the $n$-sphere," Acta Arithmetica, vol. 91, no. 3, pp. 199-208, 1999.

[10] B. Osgood, R. Phillips, and P. Sarnak, "Extremals of determinants of Laplacians," Journal of Functional Analysis, vol. 80, no. 1, pp. 148-211, 1988.

[11] J. R. Quine and J. Choi, "Zeta regularized products and functional determinants on spheres," The Rocky Mountain Journal of Mathematics, vol. 26, no. 2, pp. 719-729, 1996.

[12] I. Vardi, "Determinants of Laplacians and multiple gamma functions," SIAM Journal on Mathematical Analysis, vol. 19, no. 2, pp. 493-507, 1988.

[13] A. Voros, "Spectral functions, special functions and the Selberg zeta function," Communications in Mathematical Physics, vol. 110, no. 3, pp. 439-465, 1987. 
[14] J. Choi, "Some mathematical constants," Applied Mathematics and Computation, vol. 187, no. 1, pp. 122$140,2007$.

[15] J. Choi, Y. J. Cho, and H. M. Srivastava, "Series involving the zeta function and multiple gamma functions," Applied Mathematics and Computation, vol. 159, no. 2, pp. 509-537, 2004.

[16] J. Choi and H. M. Srivastava, "Certain classes of series involving the zeta function," Journal of Mathematical Analysis and Applications, vol. 231, no. 1, pp. 91-117, 1999.

[17] J. Choi and H. M. Srivastava, "An application of the theory of the double gamma function," Kyushu Journal of Mathematics, vol. 53, no. 1, pp. 209-222, 1999.

[18] J. Choi and H. M. Srivastava, "Certain classes of series associated with the zeta function and multiple gamma functions," Journal of Computational and Applied Mathematics, vol. 118, no. 1-2, pp. 87-109, 2000, Higher transcendental functions and their applications.

[19] J. Choi, H. M. Srivastava, and V. S. Adamchik, "Multiple gamma and related functions," Applied Mathematics and Computation, vol. 134, no. 2-3, pp. 515-533, 2003.

[20] J. Choi, H. M. Srivastava, and J. R. Quine, "Some series involving the zeta function," Bulletin of the Australian Mathematical Society, vol. 51, no. 3, pp. 383-393, 1995.

[21] H. M. Srivastava and J. Choi, Series Associated with the Zeta and Related Functions, Kluwer Academic Publishers, Dordrecht, The Netherlands, 2001.

[22] J. W. L. Glaisher, "On the product $1^{1} 2^{2} \cdots n^{n}$," Messenger of Mathematics, vol. 7, pp. 43-47, 1877.

[23] J. W. L. Glaisher, "On the constant which occurs in the formula for $1^{1} 2^{2} \cdots n^{n}$," Messenger of Mathematics, vol. 24, pp. 1-16, 1894.

[24] http://mathworld.wolfram.com/Glaisher-KinkelinConstant.html .

[25] C.-P. Chen, "Glaisher-Kinkelin constant," Integral Transforms and Special Functions, IFirst, pp. 1-8, 2011.

[26] H. M. Srivastava and J. Choi, Zeta and Q-Zeta Functions and Associated Series and Integrals, Elsevier Science Publishers, Amsterdam, The Netherlands, 2012.

[27] V. S. Adamchik, "Polygamma functions of negative order," Journal of Computational and Applied Mathematics, vol. 100, no. 2, pp. 191-199, 1998.

[28] L. Bendersky, "Sur la fonction gamma généralisée," Acta Mathematica, vol. 61, no. 1, pp. 263-322, 1933.

[29] G. H. Hardy, Divergent Series, Clarendon Press, Oxford University Press, Oxford, UK, 1949.

[30] G. H. Hardy, Divergent Series, Chelsea PublishingCompany, New York, NY, USA, 2nd edition, 1991.

[31] J. Edwards, A Treatise on the Integral Calculus with Applications: Examples and Problems, vol. 1-2, Chelsea Publishing Company, New York, NY, USA, 1954.

[32] R. L. Graham, D. E. Knuth, and O. Patashnik, Concrete Mathematics: A Foundation for Computer Science, Addison-Wesley Publishing Company, Reading, Mass, USA, 2nd edition, 1994.

[33] Y.-H. Zhu and B.-C. Yang, "Accurate inequalities for partial sums of a type of divergent series," Acta Scientiarum Naturalium Universitatis Sunyatseni, vol. 37, no. 4, pp. 33-37, 1998. 


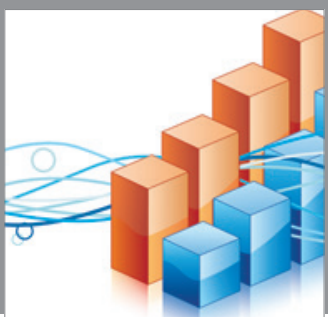

Advances in

Operations Research

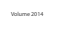

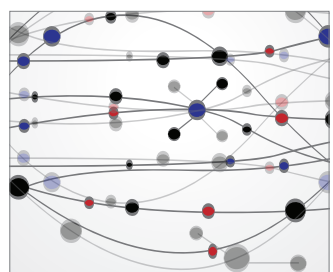

\section{The Scientific} World Journal
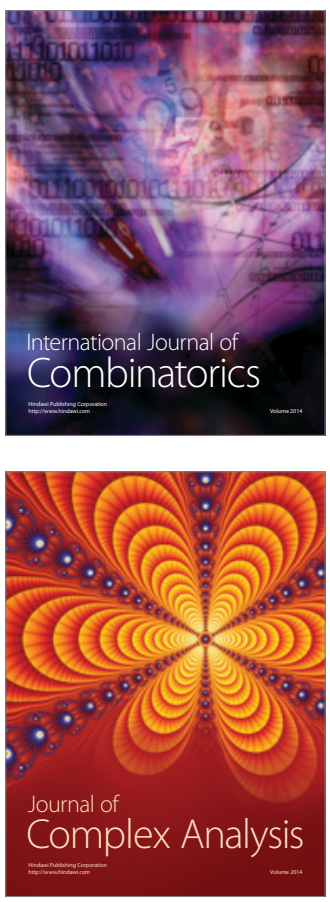

International Journal of

Mathematics and

Mathematical

Sciences
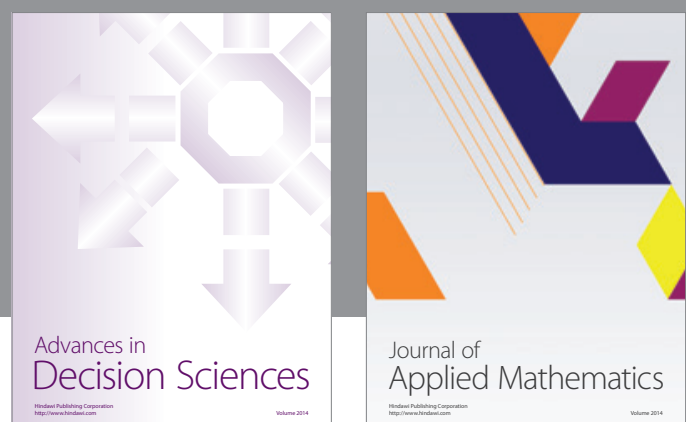

Journal of

Applied Mathematics
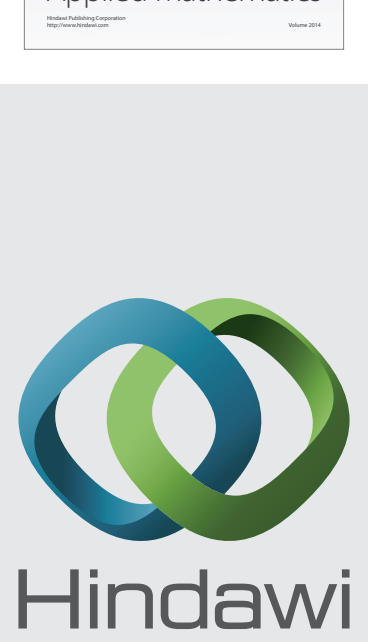

Submit your manuscripts at http://www.hindawi.com
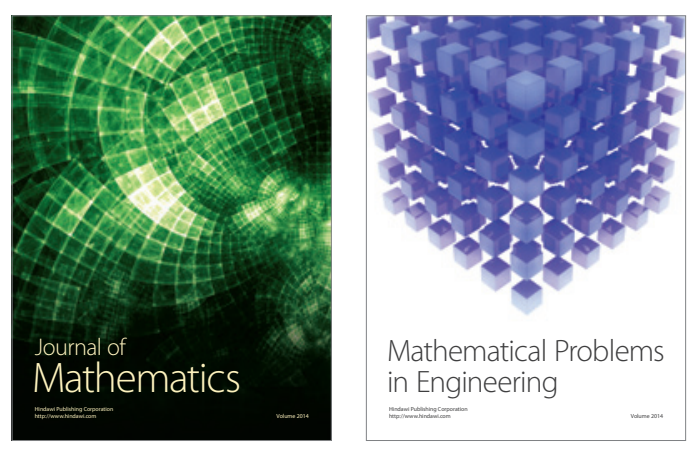

Mathematical Problems in Engineering
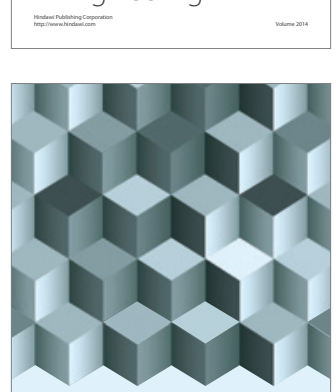

Journal of

Function Spaces
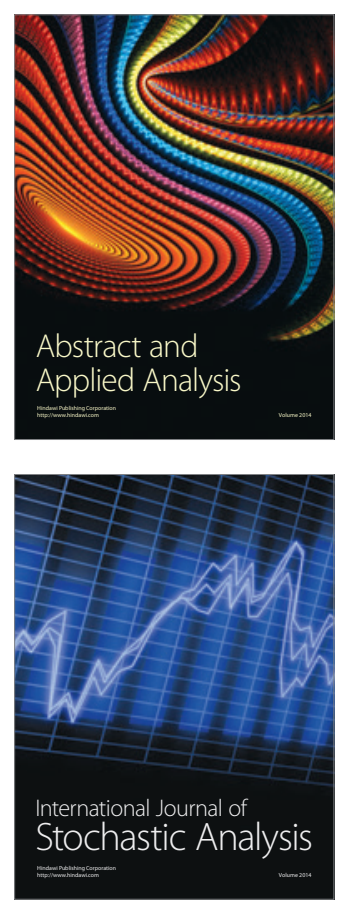

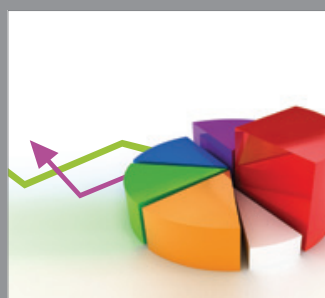

ournal of

Probability and Statistics

Promensencen
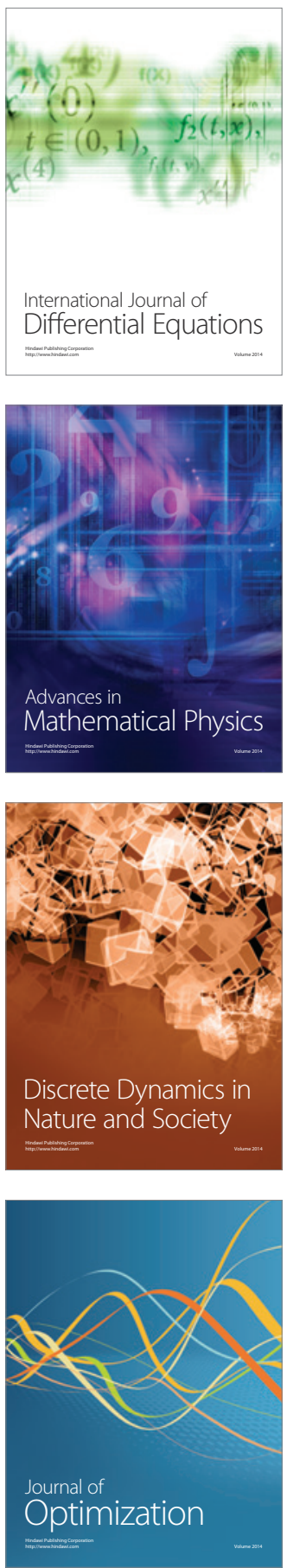